\title{
Self-Interference Cancellation Models for Full-duplex Wireless Communications
}

\author{
Andrew Thangaraj, Radha Krishna Ganti, and Srikrishna Bhashyam \\ Department of Electrical Engineering \\ Indian Institute of Technology Madras, Chennai 600036 \\ Email: \{andrew, rganti, skrishna\}@ee.iitm.ac.in
}

\begin{abstract}
In this paper, we study two models for self or loopback interference cancellation in full-duplex wireless communications. Both models are based on an underlying Z-channel with side information. We obtain achievable rate regions with suitable coding schemes under both models. Under model 1, where the self-interference channel gain is random, we employ training to estimate the unknown gain, and optimize the required training time. Under model 2, where the self-interference gain is exactly known, we show that the capacity of an ideal fullduplex node can be realized even when the side information is low rate and quantized. Our results show that loopback interference, rather than being treated as noise, can be effectively dealt with by suitable coding.
\end{abstract}

\section{INTRODUCTION}

A wireless node is a device that can receive and transmit radio frequency $(\mathrm{RF})$ signals for communications purposes in a fixed bandwidth. In most practical realizations, wireless nodes are designed to be half-duplex i.e., the node can either transmit or receive at any given time, but not both simultaneously. The reason is as follows. Suppose a node has one receive antenna and another transmit antenna. Since the receive antenna's input signals are several orders of magnitude lower than the transmit antenna's output signals, loopback interference from the transmitting antenna to the receive antenna will drown out the input signal.

Recently, work in [1][2][3] has shown practical implementations of full-duplex wireless nodes, where loopback interference is cancelled by suitable signal processing in RF and/or baseband level. A generic block diagram of such a fullduplex wireless node is shown in Fig. 1. In Fig. 1, the node is

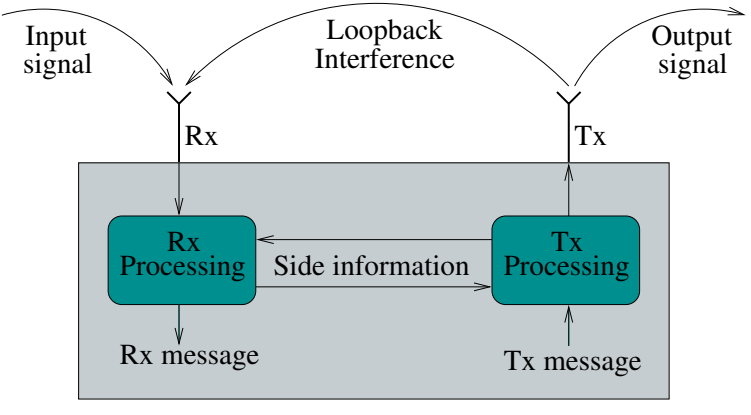

Fig. 1. Block diagram of a practical full-duplex wireless node.

internally shown to have two sub-blocks - one for transmitter processing and the other for receiver processing. The two subblocks can communicate to each other, and this is shown as the side information exchanged between the two sub-blocks.
The output signal from the transmit antenna loops back as interference to the receive antenna. In practical implementations, as reported in [2][3], exact cancellation of the loopback interference may not be feasible at the receiver sub-block, even with the use of side information from the transmitter subblock. This is because (a) the loopback interference signal may not be exactly known to either the transmitter or the receiver sub-blocks, and (b) constraints such as dynamic range, resolution of sampling circuitry and phase noise in RF circuitry might restrict the cancellation capability.

Another related area, where there has been recent interest in full-duplex communications, is the installation of indoor repeaters and relays to improve coverage [4][5]. For indoor repeaters and relays, the transmitter and receiver processing units operating in full-duplex mode could be located sufficiently far apart within a building. The model of Fig. 1 still holds for such a scenario with suitable modifications to incorporate the distance between the transmitter and receiver. We will refer to such a transmit-receive pair situated within a building as an indoor transceiver ${ }^{1}$ to distinguish from a fullduplex node. One effect of the increased distance is that the loopback interference will be lower, but the capacity of side information can also be lower.

The main goal of our work is to develop informationtheoretic models suitable for practical full-duplex communications in a wireless node and indoor transceivers with possible limits on side information. In such models, there are transmitter and receiver subnodes with a loopback link (see Fig. 2). The transmitter and receiver subnodes are assumed to be connected by side information links. In our first model applicable to full-duplex nodes with co-located antennas, we assume that the loopback gain is unknown and that the side information links are of infinite capacity. We incorporate training for the loopback channel gain, and provide achievable rate regions for independent transmission rate to a destination node and reception rate from another source node. In our second model, we consider the scenario where the loopback gain is known exactly and the side information channel has a finite capacity. Such models can be used to study coding methods and capacity of wireless networks with practically realizable full-duplex nodes and indoor transceivers.

Full-duplex wireless communications with loopback interference has been studied by several authors in recent years. A lot of interest has been shown in the study of

\footnotetext{
${ }^{1}$ We will not use the transceiver as a relay in this work. So, we have avoided the term "relay".
} 
a relay channel with a full-duplex relay node with loopback interference, and comparison with half-duplex relaying [6][7][5][8][9][10]. Signal processing methods for nulling the loopback interference in MIMO relays has also received attention [7][11][12][13][14][15]. While some of these works derive capacity under specific assumptions on the processing at the relay, information-theoretic models for the loopback interference in wireless nodes seem to have emerged in the literature only very recently [16]. In [16], the deterministic approximate capacity method has been used for a full-duplex relaying system with an unknown loopback gain. In contrast, our study uses traditional random coding methods for a fullduplex communication set-up, where a full duplex node or indoor relay is transmitting information to a second destination node and receiving independent information from a third source node. Also, our second model with a known loopback gain and limited side information capacity appears to be new in the context of full-duplex communications.

The rest of this article is organized as follows. The models for practical full-duplex communications are introduced in Section II. In Section III, these models are applied and schievable rate regions are derived in a simple setting where a full-duplex node or indoor relay is receiving information from one node, and simultaneously transmitting independent information to another node.

\section{MOdELS FOR FULL-DUPLEX COMMUNICATIONS}

In this section we introduce two models for full-duplex communication capabilities with practical constraints on cancellation of loopback interference.

A base model for a full-duplex node or indoor transceiver is shown in Fig. 2. A single full-duplex node or transceiver is

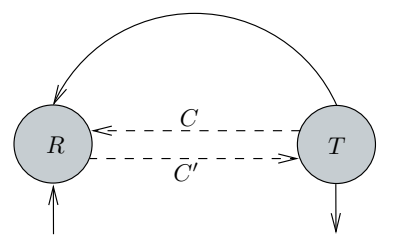

Fig. 2. Model of a practical full-duplex wireless node.

represented in Fig. 2 as a node $R$ and a node T, representing the receiving and the transmitting sub-blocks, respectively. The solid directed link from $\mathrm{T}$ to $R$ represents the loopback interference link. We will typically assume that if $X$ is transmitted by $\mathrm{T}$, a noisy version of $\delta X$ is received on the loopback link, where $\delta$ is a constant that may or may not be known to both $R$ and T.

The dotted lines in Fig. 2 represent the side information links between $R$ and $\mathrm{T}$ with capacities $C$ and $C^{\prime}$, as shown. The incoming link carrying the input signal into $R$ and the outgoing link from $\mathrm{T}$ carrying the output signal are also shown in the model. At $R$, the loopback signal and the input signal will add to form the received signal. By the broadcast nature of wireless nodes, the signal transmitted by $\mathrm{T}$ is carried on both the solid edges out of $\mathrm{T}$ as the output signal and the loopback interference. However, the side information links are independent of all other links, and could carry any independently encoded information. These capacities will be assumed to be finite or infinite depending on the model. Since $R$ and $\mathrm{T}$ are part of the same node, encoding and decoding operations can be performed at either node.

\section{A. Full-duplex communications setting}

We consider the setting where the full-duplex node or indoor transceiver $\mathrm{F}$ receives information from a node $\mathrm{A}$ and simultaneously transmits independent information to another node B. Adding nodes A and B to Fig. 2, we obtain the model in Fig. 3, which we immediately recognize as a Z-channel with side information. In Fig. $3, X_{1}$ represents the transmit symbol

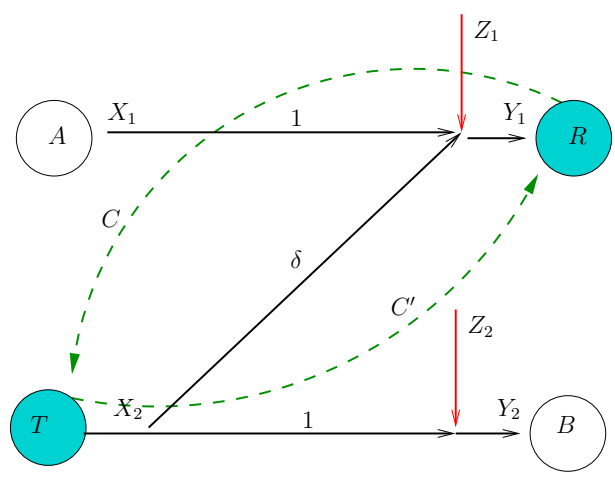

Fig. 3. The equivalent AWGN Z-channel model for practical full-duplex communications. The main difference to the conventional Z-channel is the availability of side-information. The capacity of the side-information channel from the transmitter module $\mathrm{T}$ of $\mathrm{F}$ to its receive module $R$ is denoted by $C^{\prime}$, while the capacity of the reverse side-information channel is denoted by $C$; $Z_{1}$ and $Z_{2}$ represent the AWGN noises with variance $\sigma_{1}^{2}$ and $\sigma_{2}^{2} ; \delta$ is the channel gain of the loopback interference path.

from $\mathrm{A}, X_{2}$ represents the transmit symbol from $\mathrm{F}, Z_{1}$ and $Z_{2}$ represent additive white Gaussian noise at the receivers $R$ and $\mathrm{B}$, and $\delta$ represents the gain of the loopback interference.

The following equations are immediate from Fig. 3:

$$
\begin{aligned}
& Y_{1}=X_{1}+\delta X_{2}+Z_{1}, \\
& Y_{2}=X_{2}+Z_{2} .
\end{aligned}
$$

We observe that (1) and (2) correspond to a Z-channel with side-information. So, it appears that the capacity region can be improved by viewing the system as a Z-channel and designing appropriate codes, rather than viewing the transmissions as independent and always treating $X_{2}$ as noise in (1).

\section{B. Two models: Wireless node versus indoor transceiver}

1) Full duplex wireless node: When the model of Fig. 3 is applied to a wireless node with co-located antennas, $\mathrm{T}$ and $R$ are located within the same block, we assume that the side-information channel capacities are infinite, i.e. $C=C^{\prime}=\infty$. This is a practical assumption since the receiver and the transmitter modules can be connected with a high speed interconnect. The imparity in this network is that the loopback interference channel gain $\delta$ is random and unknown to the node (both the transmit module $\mathrm{T}$ and receive module $R$ ). In the rest of this paper, this is referred to as Model 1 . 
2) Indoor transceiver: In contrast, when the model of Fig. 3 is applied to a wireless indoor transceiver with antennas separated by some distance, we assume that side-information channel capacities are finite. This is because the transceiver and the receiver modules have to be interconnected by some mechanism, for e.g., Lan or a cable. However, we will assume that the loopback interference channel gain $\delta$ is assumed to be known to both the transmit module $\mathrm{T}$ and receive module $R$. We term such a model as Model 2.

\section{Achievable Rate Regions}

In this section, we derive achievable rate regions for Models 1 and 2. More precisely, if $R_{1}$ denotes the rate of communication between $\mathrm{F}$ and $\mathrm{A}$, and $R_{2}$ the rate of communication between $\mathrm{B}$ and $\mathrm{F}$, we are interested in characterizing the rate pairs $\left(R_{1}, R_{2}\right)$ such that error free communication is possible in the standard information-theoretic sense. We denote this rate region by $\mathcal{R}_{\mathrm{F}}$. We then compare $\mathcal{R}_{\mathrm{F}}$ with the rate regions obtained when $\mathrm{F}$ is either an ideal full-duplex node or a halfduplex node. It is easy to see that $\mathcal{R}_{\mathrm{H}} \subset \mathcal{R}_{\mathrm{F}} \subset \mathcal{R}_{\mathrm{I}}$, where $\mathcal{R}_{\mathrm{H}}$ and $\mathcal{R}_{\mathrm{I}}$ are the achievable rate regions corresponding to $\mathrm{F}$ being half duplex and ideal full-duplex respectively. Hence $\operatorname{Area}\left(\mathcal{R}_{\mathrm{F}} \backslash \mathcal{R}_{\mathrm{H}}\right)$ provides a good measure of the gain obtained because of the full-duplex capabilities of the node $F$, while the quantity $\operatorname{Area}\left(\mathcal{R}_{\mathrm{I}} \backslash \mathcal{R}_{\mathrm{F}}\right)$ measures the performance loss due to the imperfections in the full-duplex implementation. We assume average power constraints on the inputs, i.e., $\mathbb{E}\left[X_{1}^{2}\right] \leq P_{1}, \mathbb{E}\left[X_{2}^{2}\right] \leq P_{2}$. Also define $F(x)=\frac{1}{2} \log _{2}(1+x)$.

\section{A. Rate region of Model 1}

Since $\delta$ is random and unknown, training is necessary to learn the gain of the self-interference path. Hence, some of the resources have to be allocated for training, which in turn leads to a reduction in the overall throughput of the system . Training is commonly used in wireless systems to estimate the channel gains. The amount of training depends mainly on the coherence time of the channel and the required precision of the estimate usually specified in terms of the mean square error. In a typical wireless system the transmitter and the receiver a priori decide on a training sequence and use this sequence to learn the channel.

In a full-duplex wireless node, the transmitter and the receiver are on the same physical device and the side information channels have infinite capacity. Therefore, the transmit data is available to the receiver instantaneously. Hence, during the training period, while the node cannot receive external information, it can transmit to an external receiver. It should also be observed that there is additional feedback from the receiver to the transmitter. During training, setting $X_{1}=0$ in (1), we get that

$$
Y_{1}=\delta X_{2}+Z_{1}
$$

where $\delta$ is the unknown gain that has to be estimated.

Let $X_{2}[k], k=1, \ldots, T$ denote the training sequence. The two sequences $X_{2}[k]$ and $Y_{2}[k]$ are known, and $\delta$ has to be estimated. A sample average of $\frac{Y_{1}[k] X_{2}[k]^{*}}{\left|X_{2}[k]\right|}$ can be used to obtain an estimate of $\delta$ with a mean square error of approximately $T^{-1}$. Let us denote such an estimate as $\hat{\delta}$. Subtracting $\hat{\delta} X_{2}$ from the RHS of (3), we get

$$
\bar{Y}_{1}=\bar{\delta} X_{2}+Z_{1}
$$

where the residual error $\bar{\delta}$ tends to a Gaussian random variable with variance $T^{-1}$. We also assume a large coherence time $T_{c}$ after which the channel might change necessitating a new estimate.

In summary, after the training, the equations for Model 1 are as follows:

$$
\begin{aligned}
& \bar{Y}_{1}=X_{1}+\bar{\delta} X_{2}+Z_{1} \\
& Y_{2}=X_{2}+Z_{2} .
\end{aligned}
$$

An achievable rate region for Model 1, as represented in (5) and (6), can be obtained by treating $\bar{\delta} X_{2}$ as noise in (1). Assuming a power constraint of $P_{1}$ to the input signal $X_{1}$ and a power constraint of $P_{2}$ for $X_{2}$, an achievable rate region with Gaussian codebooks is given by

$$
\begin{aligned}
& R_{1} \leq\left(1-\frac{T}{T_{c}}\right) \mathbb{E}\left[F\left(\frac{P_{1}}{1+\frac{X_{2}^{2}}{T}}\right)\right], \\
& R_{2} \leq F\left(P_{2}\right) .
\end{aligned}
$$

It is easy to observe that there is an optimal $T^{*}\left(T_{c}\right)$ which maximizes the rate $R_{1}$, a standard problem in non-coherent communications. See Figure 4.

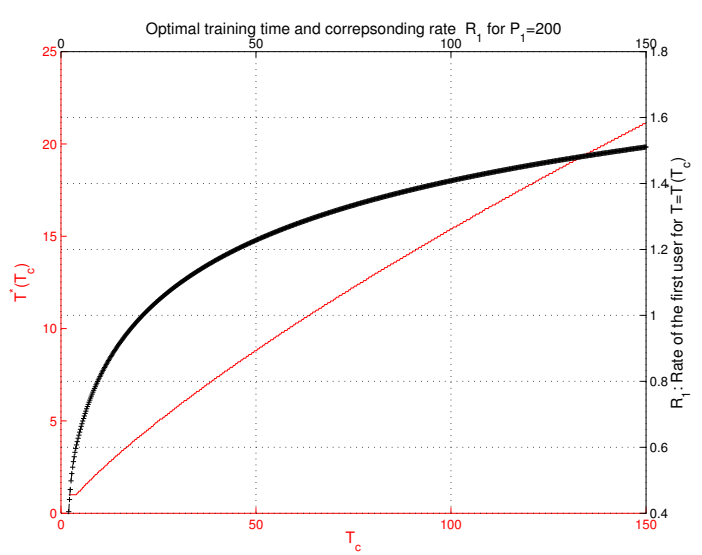

Fig. 4. The curve without markers (in red) corresponds to $T^{*}\left(T_{c}\right)$ versus $T$ for $P_{1}=200$. The other curve is the corresponding rate $R_{1}$ for the optimal threshold.

\section{B. Rate region of Model 2}

In Model 2, $\delta$ is constant and known to both the nodes $\mathrm{T}$ and $R$. We provide an achievable region for this model by neglecting the side-information from $R$ to T. Currently, even in practical full-duplex systems, this feedback is not used.

We provide the results for the discrete memoryless channel (DMC) corresponding to Fig. 3. This DMC is characterized by the probability distribution $p\left(y_{1}, y_{2} \mid x_{1}, x_{2}\right)=$ $p\left(y_{1} \mid x_{1}, x_{2}\right) p\left(y_{2} \mid x_{2}\right)$. The capacity region of this channel even without side-information is not known. Most of the known achievable schemes are based on the Han-Kobayashi achievable scheme [17] for the interference channel. We modify this 
Han-Kobayashi scheme to incorporate the side-information from $\mathrm{T}$ to $R$.

The transmitter $\mathrm{T}$ splits its codebook into two parts of rates $R_{21}$ and $R_{22}$, the common and the private information. The intended receiver $\mathrm{B}$ decodes both parts targetting a rate $R_{2}=R_{21}+R_{22}$. On the other hand, the receiver $R$ decodes information from its intended transmitter $A$ and also the part of information from $\mathrm{T}$ corresponding to rate $R_{21}$ akin to a MAC channel. We use the side-information channel to provide additional information to $R$ about the public codeword corresponding to rate $R_{21}$.

Theorem 1. Let $\mathcal{P}$ be the set of distributions of the form $p\left(x_{1}, w_{2}, u_{2}, q\right)=p\left(x_{1} \mid q\right) p\left(w_{2} \mid q\right) P\left(u_{2} \mid q\right) p(q)$. Let $\mathcal{R}_{p}$ denote the set of all rate pairs $\left(R_{1}, R_{2}\right)\left(R_{2}=R_{21}+R_{22}\right)$ satisfying the following inequalities:

$$
\begin{aligned}
R_{1} & \leq I\left(X_{1} ; Y_{1} \mid U_{2}, Q\right), \\
R_{21} & \leq I\left(U_{2} ; Y_{1} \mid X_{1}, Q\right)+C^{\prime}, \\
R_{1}+R_{21} & \leq I\left(X_{1}, U_{2} ; Y_{1} \mid Q\right)+C^{\prime}, \\
R_{21} & \leq I\left(U_{2} ; Y_{2} \mid W_{2}, Q\right), \\
R_{22} & \leq I\left(W_{2} ; Y_{2} \mid U_{2}, Q\right), \\
R_{21}+R_{22} & \leq I\left(U_{2}, W_{2} ; Y_{2} \mid Q\right) .
\end{aligned}
$$

Then any rate pair in the closure of $\cup_{p \in \mathcal{P}} \mathcal{R}_{p}$ is achievable under Model 1.

Proof: When $C^{\prime}=0$, the above rate region corresponds to the Han-Kobayashi region for the Z-channel. We do the following to utilize the side-information:

1) Encoding: Encoding is exactly the same as in HanKobayashi achievability scheme.

2) Random binning: Each of the $2^{n R_{21}}$ codewords corresponding to the public codebook is randomly assigned an integer from the set $\left\{1,2, \ldots, 2^{n C^{\prime}}\right\}$. Denote the set of codewords which are assigned integer $i$ by $S_{i}$. The sets $S_{i}$ are revealed apriori to $R$.

3) The transmitter sends the integer corresponding to the public codebook using the side-information channel of capacity $C^{\prime}$.

4) As in Han-Kobayashi achievability scheme, the decoder at $R$, does a joint typicality decoding of $\left(Y_{1}, X_{1}, U_{2}\right)$, where $U_{2}$ corresponds to the public codebook. In our case, the decoder takes the intersection of the sequences $\left(X_{1}, U_{2}\right)$ which are jointly typical with $Y_{1}$ and the set $S_{k}$, where $k$ is the integer associated with the transmitted public information.

At $R$, since the side information is used to remove the ambiguity of public message it can easily shown that the rate of the public codebook can be increased by $C^{\prime}$ compared to the standard Han-Kobayashi scheme.

Using Fourier-Motzkin elimination [18], the region $\mathcal{R}_{p}$ can be simplified to

$$
\begin{aligned}
R_{1} & \leq I\left(X_{1} ; Y_{1} \mid U_{2}, Q\right), \\
& R_{2} \leq I\left(U_{2}, W_{2} ; Y_{2}, Q\right), \\
& R_{2} \leq I\left(U_{2} ; Y_{2} \mid W_{2}, Q\right)+I\left(W_{2} ; Y_{2} \mid U_{2}, Q\right), \\
R_{2} & \leq I\left(W_{2} ; Y_{2} \mid U_{2}, Q\right)+I\left(U_{2} ; Y_{1} ; X_{1}, Q\right)+C^{\prime}, \\
R_{1}+R_{2} & \leq I\left(U_{2}, X_{1} ; Y_{1} \mid Q\right)+I\left(W_{2} ; Y_{2} \mid U_{2}, Q\right)+C^{\prime} .
\end{aligned}
$$

In the above inequalities, $Q$ is the time sharing parameter. We now evaluate the inequalities when the DMC is replaced with an AWGN channel with power constraints and for a time sharing variable that takes a single value $Q=\phi$ with probability one. The AWGN channel model is given by

$$
\begin{aligned}
& Y_{1}=X_{1}+\delta\left(U_{2}+W_{2}\right)+Z_{1}, \\
& Y_{2}=U_{2}+W_{2}+Z_{2},
\end{aligned}
$$

where $Z_{1}$ and $Z_{2}$ are unit variance independent Gaussian random variables. In particular, observe that we have assumed $X_{2}=U_{2}+W_{2}$, although the rate splitting can be done in any arbitrary way. We split the input power of $X_{2}$ as $\mathbb{E}\left[U_{2}^{2}\right] \leq \lambda P_{2}$ and $\mathbb{E}\left[W_{2}^{2}\right] \leq(1-\lambda) P_{2}$, where $0 \leq \lambda \leq 1$. Then the pentagonal region given by inequalities in Theorem 1 equals

$$
\begin{aligned}
R_{1} & \leq F\left(\frac{P_{1}}{1+\delta^{2}(1-\lambda) P_{2}}\right), \\
R_{2} & \leq F\left(P_{2}\right), \\
R_{2} & \leq F\left(\frac{\delta^{2} \lambda P_{2}}{1+\delta^{2}(1-\lambda) P_{2}}\right)+F\left(1+(1-\lambda) P_{2}\right)+C^{\prime}, \\
R_{1}+R_{2} & \leq F\left(\frac{P_{1}+\delta^{2} \lambda P_{2}}{1+\delta^{2}(1-\lambda) P_{2}}\right)+F\left(1+(1-\lambda) P_{2}\right)+C^{\prime} .
\end{aligned}
$$

It is well known that if $\delta>1$, then the Han-Kobayashi scheme is optimal, i.e., achieves capacity. However, this region might be less than the capacity region achieved without interference. The next corollary characterizes the required rate of side information for the node to act as an ideal-full duplex node.

Corollary 1. The capacity of the full-duplex node with limited side-information equals the capacity of an ideal full duplex node if the rate of the feedback channel from $\mathrm{T}$ to $R$

$$
C^{\prime} \geq C^{*}=F\left(P_{1}\right)+F\left(P_{2}\right)-F\left(P_{1}+\delta^{2} P_{2}\right),
$$

and is achieved by setting $\lambda=1$.

Proof: We first choose $\lambda=1$. The proof follows by setting $C^{\prime}$ such that

$$
F\left(P_{1}\right)+F\left(P_{2}\right)=F\left(P_{1}+\delta^{2} P_{2}\right)+C^{\prime},
$$

and observing that the region $R_{1} \leq F\left(P_{1}\right)$ and $R_{2} \leq F\left(P_{2}\right)$ can be achieved.

Observe that $C^{*}<F\left(P_{2}\right)$, i.e., the side-information channel can have a capacity that is less than the maximum transmission rate of the T to $B$ link. From a design perspective, it implies that the capacity of an ideal-duplex node can be realized, even when the side information is low rate and quantized (or non ideal).

In Fig. 5, the achievable rate regions for the AWGN Zchannel are presented for $C^{\prime}=1.2$ and $C^{\prime}=3.6$. Using 
Corollary 1 , we obtain $C^{*} \approx 0.77$ for this configuration. The right figure in Fig. 5 correspond to $C^{\prime}=3.6>C^{*}$ and hence the achievable region coincides with that of an ideal full-duplex node. We also observe that the achievable regions are always larger than those corresponding to a half-duplex node.
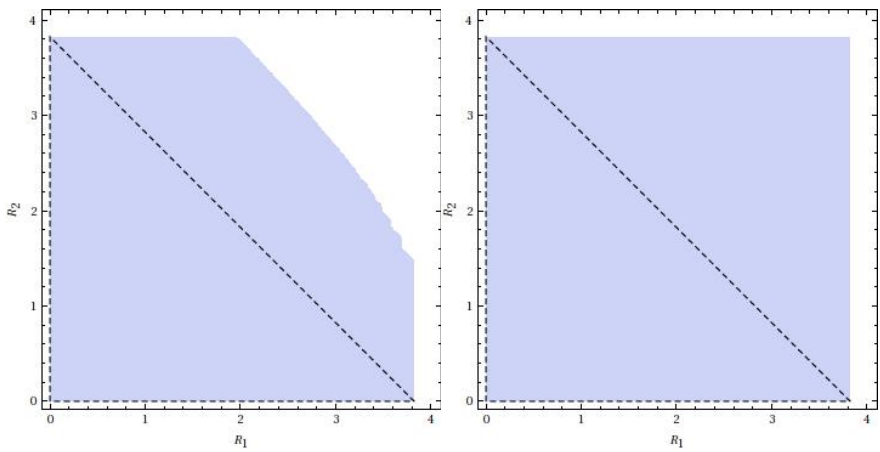

Fig. 5. Illustration of achievable rate regions for the AWGN Z-channel with side-information. The dark region corresponds to the rate region of the full-duplex node with the modified Han-Kobayashi achievablity scheme. The region within the dashed curve corresponds to the achievable rate region of a half-duplex node. We have chosen $P_{1}=P_{2}=200, \delta=0.7$. The left figure corresponds to $C^{\prime}=1.2$ and the right figure corresponds to $C^{\prime}=$ $3.6>C^{*} \approx 3.54$. The jagged edges are because of numerical limitations in computing unions of several pentagonal regions.

\section{Relating Model 1 with Model 2}

We now provide a heuristic relationship between both the models. Observe that $T^{-1}$ in Model 1 denotes the ambiguity in knowing the gain of the self interference loop. This ambiguity results in interference which cannot be removed. Also for this model, a capacity achieving scheme is to treat this selfinterference as noise. Hence, while not exact, we can consider the following set of input-output equations for Model1.

$$
Y_{1}=X_{1}+X_{2}+N_{2},
$$

with side-information $Y_{s}=X_{2}+N_{s}$ where $N_{s} \sim N\left(0, \frac{P_{2}}{T}\right)$. The receiver can now subtract $Y_{s}$ from $Y_{1}$ resulting in "almost" the same capacity given by (7).

Now it is easy to relate this model with Model 2 which has limited rate side-information. Using rate-distortion theory, it is easy to see that $C^{\prime}$ should be at least $\frac{1}{2} \log \left(P_{2} /\left(P_{2} / T^{*}\left(T_{c}\right)\right)\right)$ so that the distortion in the side information is less than $\epsilon P_{2}$. So Models 1 and 2 can be related by setting $C^{\prime}=$ $\frac{1}{2} \log \left(T^{*}\left(T_{c}\right)\right)$. In Fig. 6, we plot the rate regions obtained by Model 1 and Model 2.

\section{REFERENCES}

[1] M. Duarte and A. Sabharwal, "Full-duplex wireless communications using off-the-shelf radios: Feasibility and first results," in Signals, Systems and Computers (ASILOMAR), 2010 Conference Record of the Forty Fourth Asilomar Conference on, nov. 2010, pp. 1558 -1562.

[2] A. Sahai, G. Patel, and A. Sabharwal, "Pushing the limits of full-duplex: Design and real-time implementation," Rice University, Houston, USA, Tech. Rep. TREE1104, Feb. 2011.

[3] J. I. Choi, M. Jain, K. Srinivasan, P. Levis, and S. Katti, "Achieving single channel, full duplex wireless communication," in Proceedings of the sixteenth annual international conference on Mobile computing and networking, ser. MobiCom '10, 2010, pp. 1-12.

[4] http://www.qualcomm.com/media/documents/, "3G Personal Repeaters," Qualcomm Inc., Tech. Rep., 2010.
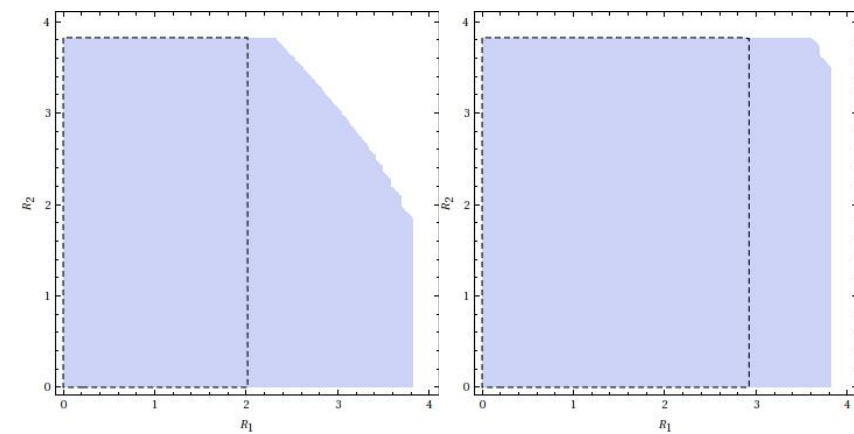

Fig. 6. The dark region corresponds to the capacity region of Model 2, while the region within the dashed curve corresponds to Model 1 . We have chosen $P_{1}=P_{2}=200, \delta=0.7$. The left figure corresponds to $T_{c}=50$ with a corresponding $T^{*}\left(T_{c}\right) \approx 8.8$ and the right figure corresponds to $T_{c}=1000$ with $T^{*}\left(T_{c}\right) \approx 86.7$. For both figures $C^{\prime}=1 / 2 \log \left(T^{*}\left(T_{c}\right)\right)$. The jagged edges are because of numerical limitations in computing unions of several pentagonal regions.

[5] K. Haneda, E. Kahra, S. Wyne, C. Icheln, and P. Vainikainen, "Measurement of loop-back interference channels for outdoor-to-indoor fullduplex radio relays," in Antennas and Propagation (EuCAP), 2010 Proceedings of the Fourth European Conference on, april 2010, pp. 1 -5 .

[6] T. Riihonen, S. Werner, and R. Wichman, "Hybrid full-duplex/halfduplex relaying with transmit power adaptation," Wireless Communications, IEEE Transactions on, vol. 10, no. 9, pp. 3074 -3085, september 2011.

[7] — "Mitigation of loopback self-interference in full-duplex mimo relays," Signal Processing, IEEE Transactions on, vol. PP, no. 99, p. 1, 2011.

[8] D. Michalopoulos, J. Schlenker, J. Cheng, and R. Schober, "Error rate analysis of full-duplex relaying," in Waveform Diversity and Design Conference (WDD), 2010 International, aug. 2010, pp. 000165 000168.

[9] T. Kwon, S. Lim, S. Choi, and D. Hong, "Optimal duplex mode for df relay in terms of the outage probability," Vehicular Technology, IEEE Transactions on, vol. 59, no. 7, pp. 3628 -3634, sept. 2010.

[10] H. Ju, E. Oh, and D. Hong, "Improving efficiency of resource usage in two-hop full duplex relay systems based on resource sharing and interference cancellation," Wireless Communications, IEEE Transactions on, vol. 8, no. 8, pp. $3933-3938$, august 2009.

[11] B. Chun and Y. H. Lee, "A spatial self-interference nullification method for full duplex amplify-and-forward mimo relays," in Wireless Communications and Networking Conference (WCNC), 2010 IEEE, april 2010, pp. $1-6$.

[12] C.-H. Lee, J.-H. Lee, Y.-W. Kwak, Y.-H. Kim, and S.-C. Kim, "The realization of full duplex relay and sum rate analysis in multiuser mimo relay channel," in Vehicular Technology Conference Fall (VTC 2010Fall), 2010 IEEE 72nd, sept. 2010, pp. 1 -5.

[13] P. Lioliou, M. Viberg, M. Coldrey, and F. Athley, "Self-interference suppression in full-duplex mimo relays," in Signals, Systems and Computers (ASILOMAR), 2010 Conference Record of the Forty Fourth Asilomar Conference on, nov. 2010, pp. $658-662$.

[14] Y. Y. Kang and J. H. Cho, "Capacity of mimo wireless channel with full-duplex amplify-and-forward relay," in Personal, Indoor and Mobile Radio Communications, 2009 IEEE 20th International Symposium on, sept. 2009 , pp. $117-121$.

[15] J. Sangiamwong, T. Asai, J. Hagiwara, Y. Okumura, and T. Ohya, "Joint multi-filter design for full-duplex mu-mimo relaying," in Vehicular Technology Conference, 2009. VTC Spring 2009. IEEE 69th, april 2009, pp. $1-5$.

[16] E. Everett, D. Dash, C. Dick, and A. Sabharwal, "Self-interference cancellation in multi-hop full-duplex networks via structured signaling," in Communication, Control, and Computing (Allerton), 2011 49th Annual Allerton Conference on, sept. 2011, pp. 1619 -1626.

[17] T. Han and K. Kobayashi, "A new achievable rate region for the interference channel," Information Theory, IEEE Transactions on, vol. 27, no. 1 , pp. $49-60$, jan 1981.

[18] H. Chong, M. Motani, H. Garg, and H. El Gamal, "On the HanKobayashi region for the interference channel," Information Theory, IEEE Transactions on, vol. 54, no. 7, pp. 3188-3195, 2008. 